\title{
Index
}

Academic voice, 35

Amusement, 157

Analytical autoethnography, 33-34, 68-69, 151

analytical to evocative autoethnography, 180

connected and disconnected emotion, 173-174

deep awe/numinousity, 174-175

descriptive model, 178-179

emotional connection, 169-171

emotional disconnection, 171

evocative autoethnographies, 151-152

graphic models, 175-178

grounded theory, 152-153

inferred emotions, 164-169

keywords and themes, 162-164

lonely/alone, 172-173

reflective or reflexive analysis, 156-160

text analysis, 153-156

theoretical models, 175

in travel and tourism, 53-57

travel emotions, 161

Analytical models, 203-205

Analytical process, 157

Anger, 16, 158

Animals of Rajpur, 117-120

Atom theory, 139

Attention deficit hyperactivity disorder (ADHD), 34-35

Attraction approach, 7

Autoethnographic/autoethnography, $18,22,76,161$

analytical autoethnography in travel and tourism, 53-57 assessing, 41-43, 198

becoming autoethnographic researcher, 193-197 case for autoethnography in tourism research, 200

criticisms, challenges and concerns, $37-41$

criticisms of and assessing, 71-72

development as research method, 23-25

early travel and tourism, 59-61

evocative, performance or analytical, 25-34

evocative travel and tourism, 57-59

final digression, 201

Flâneusing, 21-23

illustrating power of, 34-37

in leisure, 45-47

in leisure, travel and tourism, 69-70

quality of research, 198-200

quality of travel and tourism, 61

reflexive analysis of methodological process, 197-198

researcher, 193-197

texts, 22

and tourism, 68-70

touristic journeys, 61-63

in travel and tourism, 47-53

warning, 200-201

Awe, 157

Basic emotions, 16

Black Feminist Autoethnography

$$
\text { (BFA), } 36
$$

Boundaries, 25

Business approach, 7

Child and traveller, 104-105

Chôra, 13

Chorister, 13

Cinema et Turismo, 60 
Coherent, 155

Cohesion, 155

Collaborative autoethnography, 58-59

Colonizing tourist-cum-researcher, 19

Confessional tale, 23

Confused/conflicted, 173

Connected emotion, 173-174

Connectedness, 177-178

Contempt, 158

Content analysis, 154-155

Continuum, 175-177

Critical analysis, 12-13

Culture, 76-78

skin of, 81-82

Data, 199

Deep awe/numinousity, 174-175

Definitional approach, 6

Depth, 66

Descriptive model, 178-179, 205

Differential Emotions Scale (DES), 10

Digressions, 2, 65

Disconnected emotion, 173-174

Disgust, 16, 158

'Ellis' approach, 38

Embarrassment, 158

Emodiversity, 11, 157, 159

Emotional connection, 169 to culture, 170

to land, 170-171

to past, 171

to people, 169-170

Emotional disconnection, 171

Emotional engagement, 56

Emotional experiences, 84 tourist experience as, $8-13$

Emotional themes and words, 163

Emotional tourist, 16-20, 203, 205

Emotions, 5, 11-12, 87 qualities, 9

role in tourist experience, 208-210 in tourism, 12

Ethical approach, 199

Ethics of autoethnography, 40-41

Evaluative Tradition, 9
Evocative autoethnography, 26-29, 68-69, 151-152, 205-208

analytical to, 180

outcomes, 181-189

storytelling, 84

Evocative travel, 57-59

Experiencescapes, 7-8, 66

Fascinans, 19, 166

Fear, 16, 158

Feeling Tradition, 9

Flâneur, 21

Flâneuse, 21

Frisson, 124-125

12 Frisson, Shivers and Slivers, 156

Ganga, 102

Ganga Arti, 102-103

Graphic models, 175, 204-205

connectedness, 177-178

continuum, 175-177

Gratitude, 157

Great Wave off Kanagawa, 123

Grounded theory, 152-153

Guest, 93-97

Guilt, 158

Happiness, 16

Hate, 158

Hierarchy of Needs, 6

Hope, 157

Humanity, 66

Immediate approach, 7

India

trauma and healing in, 117-120

traveling experience in, 88-89

Individual tourist experience, 6

Inferred emotional themes, 164

Inferred emotions, 158-159, 164-169

Inspiration, 157

Interest, 157

Iterative journey, 158 
Japan, traveling experience in, 89-91

Jerusalem Syndrome, 19

Journaling, 47

Journey modes, 6

Joy, 17, 158

Kanji characters, 106

Katsuobushi, 115-116

Key-themes-in-context, 164

Keywords-in-context, 156, 158

Kumbh Mela, 102

Leisure, autoethnography in, 45-47, 69-70

Literary autoethnography, 23

Lonely/alone, 172-173

Love, 158

Man from Snowy River, The (Australian classic poem), 78

Marwari Horse, 98-99

Memorable experiences, 16

Memory

and recall, 66-67

role and recall in tourist work, 15

Messiness, 12

Metanoia, 140

Modes of journey, 6

Modes of tourist experiences. 65

Monoamine neurotransmitters, 12

Motivational Tradition, 9

Mysterium, 19, 166

Nakasendo Way, 97-98

Negative emotions, 19-20

Neuroscience, 12

Non-governmental organisation

(NGO), 127

Objectivity, 23

Omotenashi, 95

Open coding, 153

Peak experiences, 18

Pedagogical leaning, 151
Performance autoethnography, 29-33, 68-69

Personal research development, 195-196

Phases of individual tourist experience, 6

Poetic tourist, 52

Post hoc satisfaction approach, 7 surveys and recollection, 11

Power of stories, 199

Pre-COVID-19 world, 1

Pride, 158

Qualities of emotion, 9

Quality of research, 198-200

Quality of travel and tourism autoethnographies, 61

Reflection, 156

Reflective analysis, 156-160

Reflexive analysis, 156-160

Reflexivity, 156, 197

Reliability, 23 of memory, 14

Relief Riders International (RRI), 80,107

Religious diversity, 81

Responsibility, 198-199

Road Trip, 5

Sacred travels, 99-104

Sadhus (holy men), 111

Sadness, 16, 158

Seeker model, 178-179

Sense of place, 12

Serenity, 158

Shame, 158

Shiokatsuo, 116

Shivers, 124-125

Slivers, 124-125

Sociocultural meanings analysis, 199

Solo travelling, 83

Spiritual rejuvenation, 84

Spirituality, 179

Stress, 158 
Surprise, 16

Systematic Self-Observation (SSO), 33

\section{Temiyage, 95}

Text analysis, 153

content analysis, 154-155

thematic and contextual identification, $155-156$

Theoretical modelling, 159-160

Theory, 59

Tourism, 10

analytical autoethnography in, 53-57

marketing, 7

Tourism Autobiographical Memory

Scale (TAMS), 14-15, 67

Tourism autoethnography, 47-53, 68-70 (see also Analytical autoethnography)

early travel and tourism, 59-61

evocative travel and tourism, 57-59

quality of travel and, 61

Tourism Recreation Research, 60

Tourist emotions, 67-68

Tourist experience, $2,5-8$ as emotional experience, $8-13$

emotional tourist, $16-20$

and emotions, 65

memory and recall, 13-16, 66-67

tourist emotions, 67-68

Trauma and healing in India, 117-120

Travel, 1,10

analytical autoethnography in, 53-57

autoethnography in, 47-53, 69-70

emotions, 161

modes, 6

quality of, 61
Traveling experience

aloha, 136-138

animals of Rajpur, 117-120

child and traveller, 104-105

creating impossible places, $128-131$

crooked angle, 88

experience of Indian wedding, 109-114

frisson, shivers and slivers, 124-125

grounding emotions, $138-140$

guest, 93-97

ill-health through overwork, 114-115

India, 88-89

India's last lesson, 133-136

Japan, 89-91

Katsuobushi, 115-117

lies, 131-133

looking from crooked angle, 91-92

Marwari Horse, 98-99

Nakasendo Way, 97-98

oblique obsessions, 120-124

ready to scream, 105-109

sacred travels, 99-104

'They Came from the Stars', $125-128$

beyond tired, 92-93

Traveller, child and, 104-105

Travelling, solo, 83

Tremendum, 19, 166

Uluru, 9-13

Validity, 23

Visual autoethnography, 52

Word repetitions, 155

World War II (WWII), 23 